Supporting Information for:

\title{
Effect of Boron Doping on the Wear Behavior of the Growth and Nucleation Surfaces of Micro and Nanocrystalline Diamond Films
}

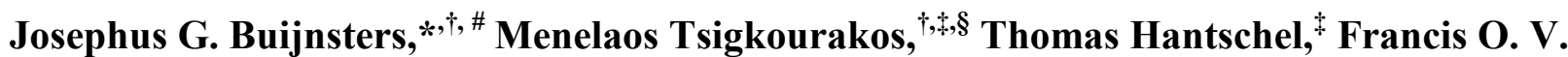 \\ Gomes, ${ }^{\ddagger} \S$ Thomas Nuytten, ${ }^{\ddagger}$ Paola Favia, ${ }^{\ddagger}$ Hugo Bender, ${ }^{\ddagger}$ Kai Arstila, ${ }^{\ddagger}, \xi$ Jean-Pierre Celis ${ }^{\#}$ \\ and Wilfried Vandervorst \\ ${ }^{\dagger}$ Department of Precision and Microsystems Engineering, Research Group of Micro and Nano \\ Engineering, Delft University of Technology, Mekelweg 2, 2628 CD Delft, The Netherlands \\ Imec, Kapeldreef 75, B-3001 Leuven, Belgium \\ ${ }^{\S}$ IKS-Department of Physics, KU Leuven, Celestijnenlaan 200D, B-3001 Leuven, Belgium \\ ${ }^{\xi}$ Department of Physics, University of Jyväskylä, P.O. Box 35, FI-40014 University of \\ Jyväskylä, Finland \\ \#Department of Materials Engineering, KU Leuven, Kasteelpark Arenberg 44, B-3001 Leuven, \\ Belgium
}

Corresponding Author contact information

*E-mail: J.G.Buijnsters@tudelft.nl.

Phone: +31 (0)15 2785396 

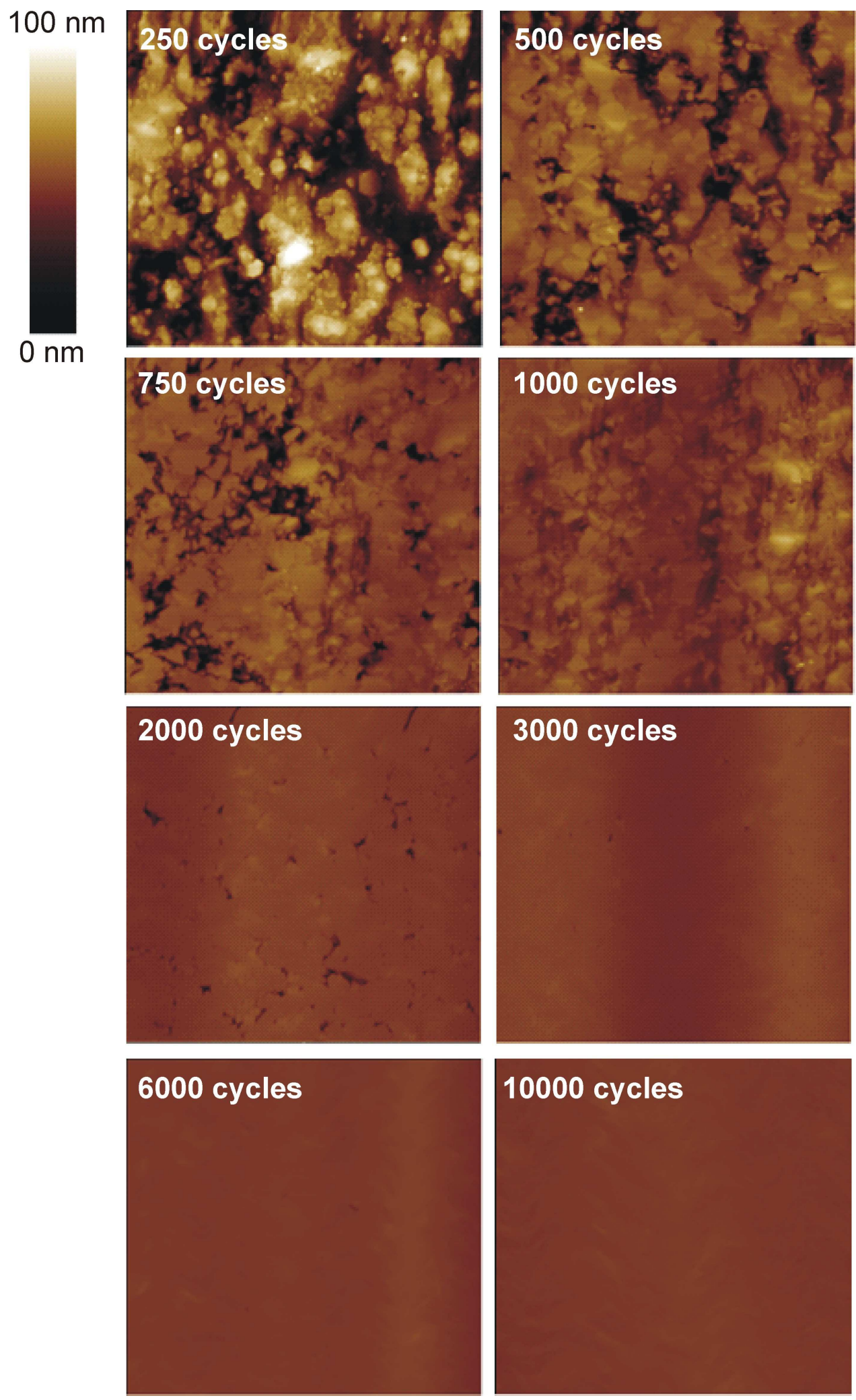

Figure S1. Topographic tapping mode AFM images taken from the centers of the wear tracks formed on the NCD1 film (2.8 at.\% B-doping level) after sliding for 250, 500, 750, 1000, 2000, 3000,6000 and 10000 cycles at $2 \mathrm{~N}$ normal load. 


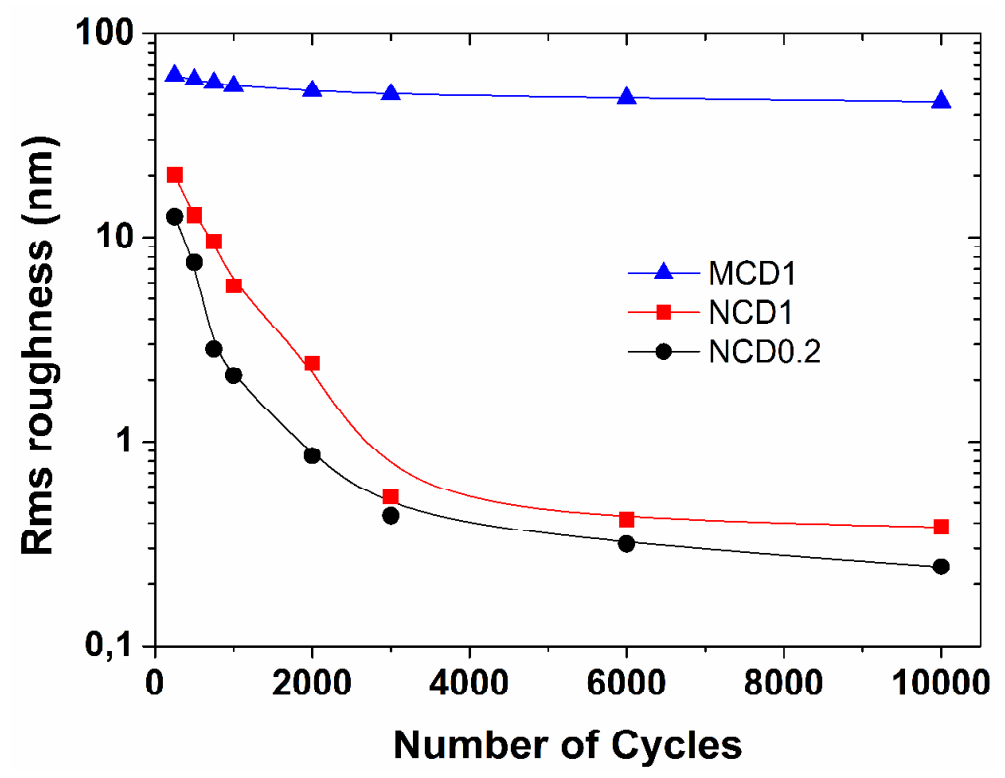

Figure S2. Comparison of the root-mean-square (rms) roughness at the center of the wear track vs. number of sliding cycles for the NCD1 (2.8 at.\% B-doping level), NCD0.2 (2.8 at.\% Bdoping level) and MCD1 (0.6 at.\% B-doping level) films. A normal load of $2 \mathrm{~N}$ was applied. The relative errors in roughness data of about $10 \%$ have been left out for reasons of figure clarity.

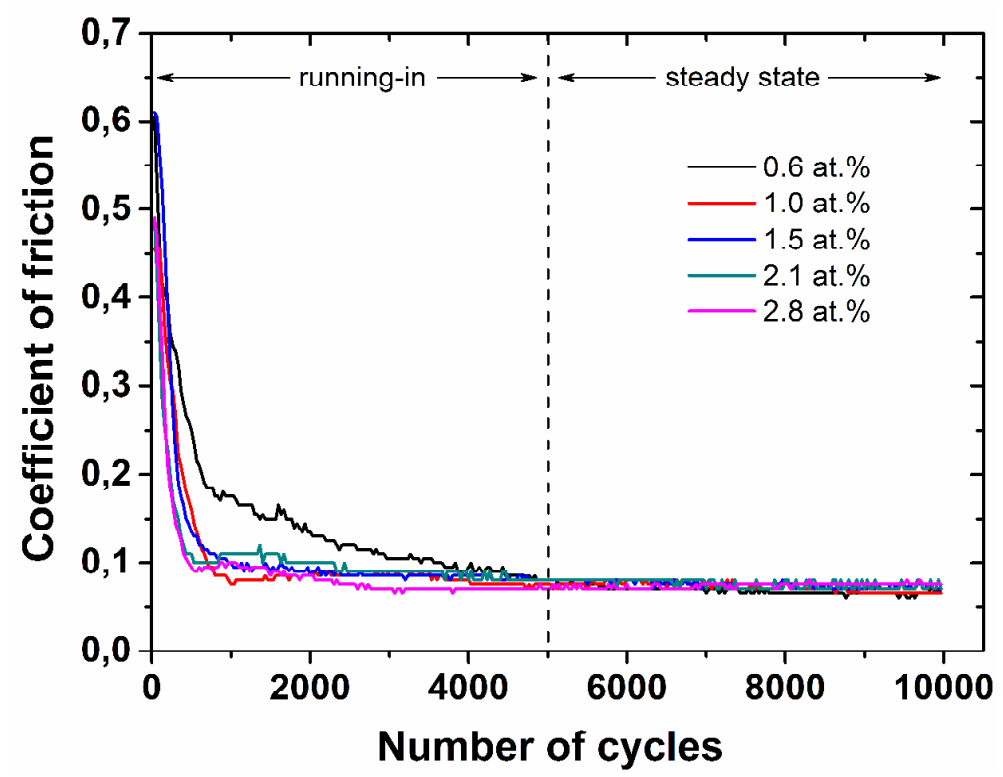

Figure S3. Coefficient of friction (COF) vs. number of sliding cycles for the NCD1 films with different B-doping levels. The vertical dashed line at 5000 cycles indicates the transition between the running-in stage and steady state sliding condition for the film with lowest B-doping level (i.e., 0.6 at.\% B). Note that steady-state sliding in the case of the films with higher B-doping levels is already obtained after only about 2000 cycles. 


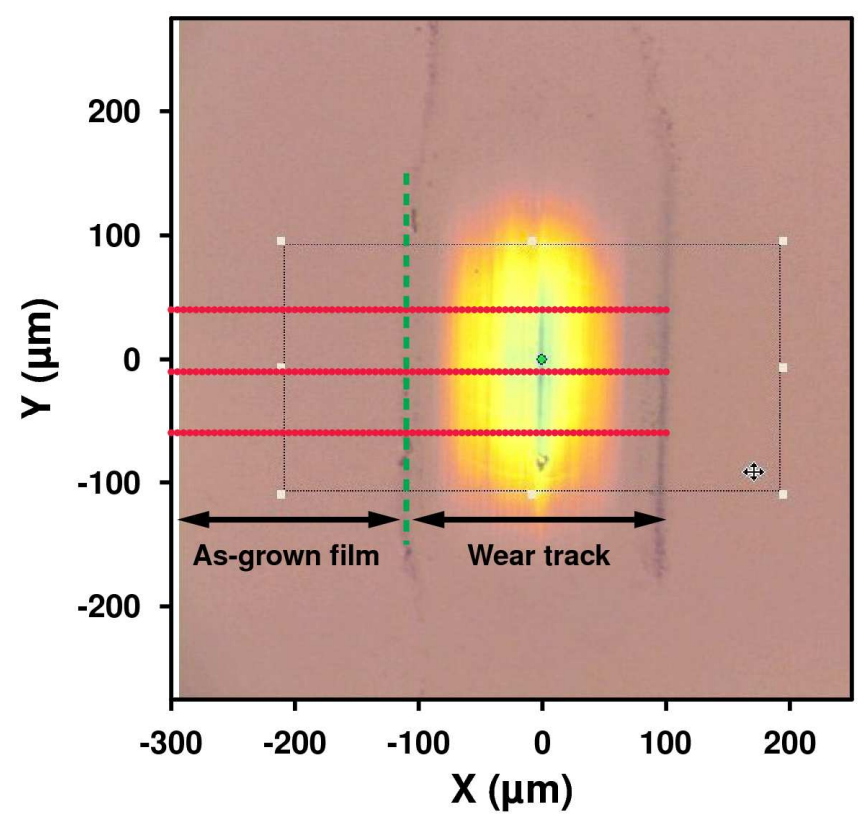

(a)

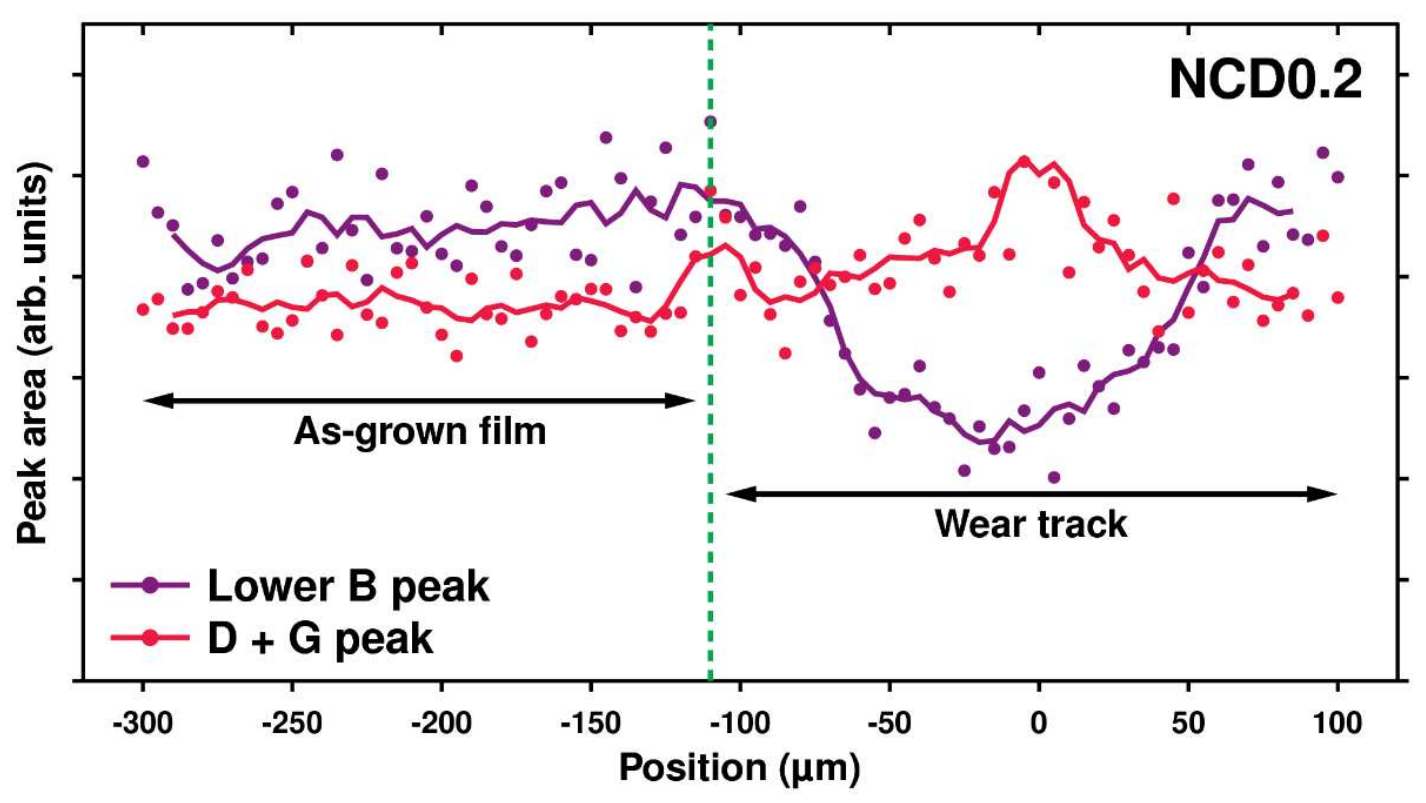

(b)

Figure S4. (a) Optical microscope image of the wear track formed on the NCD0.2 film (2.8 at.\% B-doping level) after 10,000 sliding cycles at $2 \mathrm{~N}$ normal load showing the Raman scan lines marked in red and (b) the peak intensities of the same illustrating the lower B peak and the D and $\mathrm{G}$ peaks as a function of position on the wear track. Lines in (b) represent five-point averages of the measurement points. 